\title{
Cultural Sensitivity in ATOD Agencies: Administrator and Staff Perceptions in the Hispanic Heartland
}

\author{
David R. Hodge \\ Paul Cardenas \\ Harry Montoya
}

\begin{abstract}
Administrator and staff perceptions $(N=72)$ of alcohol, tobacco, and other drugs (ATOD) agency cultural sensitivity were explored in a predominantly Hispanic rural area with elevated levels of acculturation and high ATOD usage. While providers generally agreed that a relatively moderate need existed for training related to cultural issues, a more nuanced picture emerged in the purview of culturally-related barriers. Administrators viewed the lack of appropriate interpreters and language as a greater barrier than did the staff. Administrators also held higher perceptions of agencies' cultural competency. The overall high assessment of cultural sensitivity may result from the substantial number of Latino providers.
\end{abstract}

Keywords: Alcohol, drugs, culture sensitivity, Hispanic, Latino, prevention, administration, ATOD agencies

$\mathrm{H}$ ispanics are projected to surpass African Americans in the next few years as the nation's largest minority group (Castex, 1994; Perez \& De La Rosa, 1993) due to their relative youth, high fertility rates, and high documented and undocumented immigration. Although long-range projections are problematic, one census estimate predicts the Hispanic/Latino population will reach 128.3 million by 2050 (Castex, 1994). Castex (1994) highlighted four social indexes as particularly salient in illuminating the Hispanic context (1992 figures): High poverty levels (26.2\% vs. $10.3 \%$ for non-Hispanic families, a high ratio of single-parent families (30\% vs. $20 \%$ for non-Hispanics), low levels of median income ( $\$ 22,848$ vs. $\$ 33,388$ for white households), and a low median age (26 vs. 33.5 years).

While it is commonly assumed that such social indicators presage high levels of alcohol, tobacco and other drugs (ATOD) usage (Austin \& Gilbert, 1989), studies exploring ATOD use and related issues have produced inconsistent results (Warheit, Vega \& Khoury, 1996). For example, the US Department of Health and Human Services' (1997) latest National Household Survey on Drug Abuse (NHSDA) data revealed overall Hispanic ATOD use and abuse as significantly below that of whites and frequently less than African Americans. It is noteworthy

David R. Hodge, M.C.S., M.S.W. is a Rene Sand Doctoral Fellow at the George Warren Brown School of Social Work, Washington University, St. Louis, Missouri, USA. Paul Cardenas, M.S.W. is President, National Multicultral Associates, Denver, Colorado. Harry Montoya, M.A. is President, Hands Across Cultures, Española, New Mexico, USA. 
that the NHSDA is the most extensive source of national information on ATOD usage patterns among members of the civilian, non-institutionalized population age 12 and over in the United States.

Conversely, other observers (Austin \& Gilbert, 1989; Caetano, 1983, 1994; Carrasquillo, 1991; Casas, et al., 1998; Deren et al., 1997; Rodriguez \& Brindis, 1995; Warheit, Vega \& Khoury, 1996; Weeks et al., 1996) have found a more nuanced picture. These authors cite, in at least some contexts, higher rates of ATOD usage and related problems as compared to the general population.

Numerous methodological factors may underlie the difference in the results obtained. Possible causes for the lack of consensus have been credited to sample size and location, non-random sampling, differences in operationalizing the constructs measured, underreporting of certain groups due to fear of deportation, undercounting youth due to the high Hispanic drop-out rate, and confounding due to the disproportionately higher rates of abstinence among Latina females (Austin \& Gilbert, 1989; Casas et al., 1998; Warheit et al., 1996). As Parker, Weaver, and Calhoun (1995) noted, despite the recent interest in exploring ATOD issues among minority populations, few studies of Latinos have been conducted.

Another critical element in the disparate findings may be the relative degree of acculturation. In aggregate, ATOD use tends to increase with acculturation (Austin \& Gilbert, 1989; Caetano, 1994; Casas et al., 1998; Deren et al., 1997; Warheit et al., 1996). For example, Warheit, Vega, and Khoury (1996) found in their longitudinal study a generally linear trend between length of time in the United States and increased substance use among all four Hispanic subgroups. Thus, high rates of recent immigration may have produced a relatively favorable portrayal of current ATOD usage in the NHSDA data set. Furthermore, this depiction may be substantially altered in the future, as a relatively young, unacculturated population is exposed to acculturating influences.

Corporate America's (Roslow \& Nicholls, 1996) recent discovery of the Hispanic market has exacerbated the acculturation effect. Alcohol and tobacco companies are leaders in employing psychologically sophisticated advertising to penetrate the Latino market (Nuiry, 1997). Examples of the manifestation of this targeted marketing effort (Rodriguez \& Brindis, 1995) are Spanish cigarette brands and the predominance of liquor outlets in Hispanic neighborhoods.

Accordingly, it seems probable that ATOD use and related issues will increase among the Latino population in the immediate future. ATOD problems are likely to grow significantly due to a high percentage of the Hispanic population reaching adolescence, an anticipated increase in the level of acculturation, and the stepped-up use of psychological marketing techniques by alcohol and tobacco advertisers. In fact, studies employing smaller sample sets of highly acculturated Hispano subjects may be more representative of the future than the extensive NHSDA study.

While consensus has not yet been reached concerning the prevalence, extent, or antecedents associated with ATOD use, a widespread agreement exists regarding the importance of developing culturally sensitive methods for delivering services to Hispanos. In addition to the importance of culture sensitivity in agency settings 
(Applewhite, Wong \& Daley, 1991; Colon, 1996; Gant \& Gutierrez, 1996; SantiagoRivera, 1995; Weeks et al., 1996), it has been cited as a critical component in a number of diverse areas. For example, assessment and prevention of aggressive behavior (Delva-Tauili'ili, 1995), health care interventions (Bird, Otero-Sabogal, Ha \& McPhee, 1996), caregiving for elders (Delgado \& Tennstedt, 1997), community organization/practice (Kahn, 1997; Weil, 1996), as well as ATOD programs (Austin \& Gilbert, 1989) have all been mentioned as areas where an awareness of Latino cultural issues is crucial.

Yet, as Gant and Gutierrez (1996) noted, the empirical investigation of agencies' cultural sensitivity is still in its infancy. While a growing body of literature that delineates the cultural characteristics significant for the delivery of services in Latino settings exists, to the best of the authors' knowledge, the cultural issues in ATOD agencies have not been empirically explored. This is a significant oversight given the growing prevalence of ATOD problems in Hispano communities.

Accordingly, this study examines cultural sensitivity issues in ATOD agencies in a predominantly Hispanic area that has experienced a high degree of acculturation. In line with Gant and Gutierrez' (1996) observations that provider perceptions of the agency environment are often more significant than the actual characteristics of the agency, administrator and staff perceptions are explored in three areas: Culturally-related training needs, culturally-oriented barriers that inhibit access to ATOD services and programs, and an overall assessment of agency cultural competency.

\section{METHOD}

As suggested above, it may be especially salient to examine perceptions in relatively acculturated environments, as such contexts may presage the future for a substantial segment of the relatively unacculturated Hispanic population. Accordingly, the geographic area surrounding the town of Española in northern New Mexico was selected. This catchment area includes the counties of southern Rio Arriba, northern Santa Fe, and southern Taos. In total, this sparsely populated district comprises an area approximately the size of Massachusetts.

In this tri-cultural area, $70 \%$ are Hispanic, with the remaining $30 \%$ equally divided between Native Americans and Anglos or non-Hispanic whites (1998 U.S. Census). Hispanics or Hispanos have been the dominant population in the area for almost 400 years. In fact, with a history of settlement stretching back to 1598, the region is the center of the nation's oldest continuous European settlement and proudly selfidentifies as the nation's Hispanic Heartland.

Despite becoming a United States possession in the mid-19th century, until relativey recently, acculturating influences have been minimal. The relative geographic and linguistic isolation, combined with the strong Spanish/Latin culture (the area was under Spanish rule for 213 years and Mexican for 25 years) and mainstream oppression (Wright, 1994), has limited acculturation. However, wide ranging societal changes dating from the end of WWII, including such factors as mass communication, increased access to transportation, and a growing influx of Anglos, have fostered a high level of acculturation in the past few decades. 
Interestingly, the area's current poverty rates, incidence of single-parent families, income levels, and median age among Hispanics approximate Castex's (1994) national averages for Latinos (New Mexico Department of Health, 1996). However, ATOD use and related problems are generally significantly higher than state or national averages (Lewis, 1997; New Mexico Department of Health, 1996). As suggested above, the rates of ATOD usage may be related to the extended acculturation process the area has experienced during the preceding 50 years.

In light of the existing ATOD problems, a number of agencies have developed programs to address the area's concerns. As part of a previous project undertaken by Hands Across Cultures - an Española-based prevention agency-an exhaustive list of the area's community agencies had been compiled. From this schedule, 52 agencies were identified as having the potential for providing ATOD services. Phone interviews with the respective organizations yielded 29 agencies involved with ATOD issues in some form (e.g., education, counseling, prevention).

After confirming ATOD services, permission was sought from each agency's executive director, CEO, or in their absence, an administrative assistant for authorization to mail surveys to the appropriate personnel. Twenty-six agencies agreed to participate. In organizations with fewer than five staff involved with the direct provision of services, every individual was mailed a survey. In agencies with 5 to 10 employees, every second staff member was sent a survey. Executive directors and when warranted by agency size, department heads or their equivalents, were sent administrator surveys.

In addition to the survey, the mailed package included a personalized cover letter, postage-paid return envelope, and a $\$ 2.00$ bill as an incentive. One week after the mailing a phone call was placed to ensure the package had arrived and to offer assistance in facilitating the return of the survey. Three weeks after the mailing a second follow-up call was placed to those individuals who had not returned completed surveys.

Thirty-two of the administrator surveys were sent to identified personnel at the 26 agencies. Twenty-four were returned, for a response rate of $75 \%$ among executive directors and department supervisors. Seventy-eight staff surveys were sent and 48 were returned, resulting in a $61 \%$ return rate by staff involved in the direct provision of ATOD services.

\section{Survey Instrument}

The instrument explored cultural issues from three angles. Administrators and staff were asked to assess their agencies in two domains: Training needs that would enhance ATOD services, and barriers that inhibit the utilization of programs and services. The questionnaire utilized a series of Likert-type scales (1=Not at all, $5=$ Extremely) to operationalize perceptions in the 10 -question training domain (see Table 2) and the 9-question barriers domain (see Table 3).

Providers were also asked to choose the best statement from a choice of four that most accurately depicted the cultural stance of their agency-the one that most accurately reflected different levels of cultural orientation (see Table 4). Researchers specializing in minority studies at Arizona State University (Castro, 1997) pretested and refined the instrument on a prior sample of administrators and staff. 


\section{FINDINGS}

\section{Sample Characteristics}

Table 1 reveals that provider characteristics are generally reflective of the surrounding community, although Native Americans are noticeably underrepresented. This finding suggests the need for further empirical exploration. The lack of Native American representation is particularly notable in light of the high percentage of Latino providers. In fact, among administrators, $71 \%$ were Hispanic (vs. $55 \%$ of staff and $70 \%$ of the catchment's general population). However, chi-square tests revealed no significant differences among provider characteristics, with the exception of professional status $\left(\chi^{2}=19, d f=3\right.$, $p<.001)$. There were, for example, substantially more social workers among staff (34\%) than administrators (8\%).

\section{Training Needs}

\begin{tabular}{|c|c|}
\hline Characteristic & $\%$ \\
\hline \multicolumn{2}{|l|}{ Gender } \\
\hline Male & 51 \\
\hline Female & 49 \\
\hline \multicolumn{2}{|l|}{ Ethnicity } \\
\hline Hispanic & 61 \\
\hline Anglo & 31 \\
\hline Native American & 4 \\
\hline Other & 4 \\
\hline \multicolumn{2}{|l|}{ Birthplace } \\
\hline New Mexico & 60 \\
\hline Southwest & 14 \\
\hline Other & 26 \\
\hline \multicolumn{2}{|l|}{ Fluency in Spanish } \\
\hline Excellent & 31 \\
\hline Good & 22 \\
\hline Fair & 39 \\
\hline None & 8 \\
\hline Social Work Degree & 25 \\
\hline \multicolumn{2}{|l|}{ Education } \\
\hline High School or $<$ & 11 \\
\hline Associates & 7 \\
\hline Bachelors & 37 \\
\hline Masters & 35 \\
\hline Doctorate & 10 \\
\hline
\end{tabular}

As Table 2 demonstrates, inde-

pendent two-tailed $t$-tests revealed no statistically significant differences between administrators and staff in any of the areas surveyed. The resulting scores of the two groups were basically congruent. The general parity is also demonstrated by the fact that administrators recorded marginally higher assessments on seven queries, while staff had slightly higher scores on the other three questions.

Table 2 is organized in descending order of perceived training need in terms of the provider mean. In other words, when administrator and staff scores are averaged together, the question concerning how to involve community members in the planning of ATOD programs received the highest score. Thus, in aggregate, providers perceived this to be the area reflecting the greatest need for training. Accordingly, it is listed first on the table. However, it should be kept in mind that there was minimal variation between the areas requiring the most and the least training. In short, providers gave all areas an essentially moderate score in terms of culturally oriented training needs.

\section{Barriers to ATOD Services}

As Table 3 reveals, there was more variation between administrators and staff in the purview of barriers. Independent two sample $t$-tests disclosed statistically sig- 


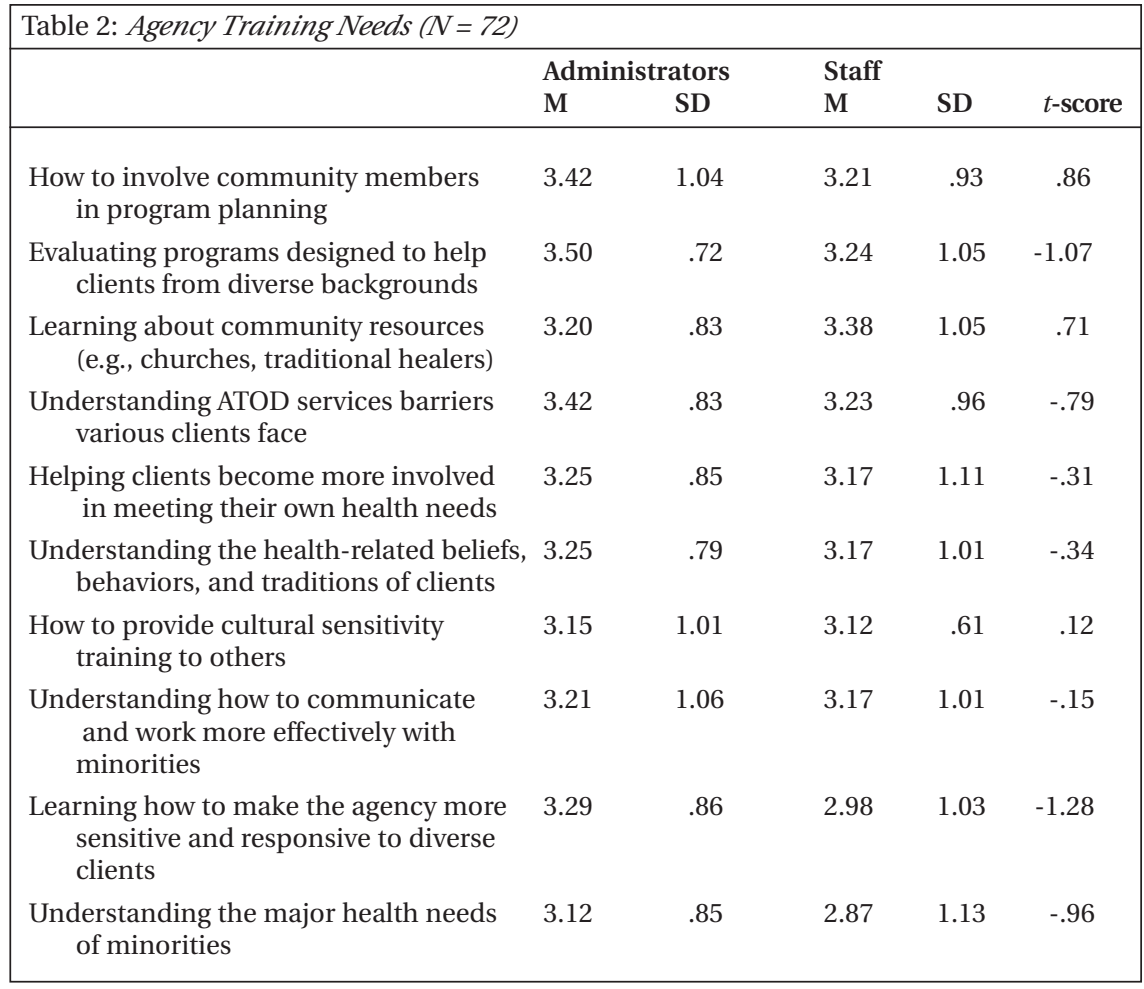

nificant differences in the areas of access to appropriate interpreters and lack of congruence between clients' language capabilities and those of providers. In both cases administrators perceived the obstacle to be of greater consequence than did the staff. However, in the remaining insignificant areas, administrators and staff were evenly split, with each assessing three areas as marginally more of an impediment.

Table 3 was also arranged in descending order based upon provider means, with the greatest obstacle listed first. Unlike training needs, which demonstrated little variation, there were marked differences in how the various barriers were rated. Client transportation was cited as the most prominent obstacle to clients receiving ATOD services, perhaps an expected finding given the poverty and lack of transportation networks in the region. Lack of advertising agency services and restrictive scheduling policies, followed by eligibility criteria, which limit some clients, were also listed as important barriers. At the other end of the continuum was the issue of appropriate interpreters and having a significant number of minority providers with whom minority clients can identify.

\section{Agencies' Cultural Competency}

Respondents were given a choice of four cultural orientations and asked to select the perspective that best embodied their agency's orientation towards cultural issues. Providers were asked to consider both program development and service 


\begin{tabular}{|c|c|c|c|c|c|}
\hline & \multicolumn{2}{|c|}{ Administrators } & \multicolumn{3}{|c|}{ Staff } \\
\hline & M & SD & M & SD & $t$-score \\
\hline Clients' limited transportation & 3.33 & 1.00 & 2.96 & 1.22 & -1.29 \\
\hline $\begin{array}{l}\text { Lack of advertising of agency services } \\
\text { in clients' communities }\end{array}$ & 2.71 & .91 & 2.41 & 1.21 & -1.06 \\
\hline $\begin{array}{l}\text { Agency's scheduling policies that don't } \\
\text { offer evening or weekend service }\end{array}$ & 2.42 & 1.21 & 2.56 & 1.16 & .48 \\
\hline $\begin{array}{l}\text { Eligibility criteria that limit access of } \\
\text { ATOD services for some groups }\end{array}$ & 2.36 & 1.25 & 2.21 & 1.12 & -.50 \\
\hline $\begin{array}{l}\text { Cultural differences between } \\
\text { clients and providers }\end{array}$ & 1.96 & .98 & 2.00 & .94 & .18 \\
\hline $\begin{array}{l}\text { Clients' language skills (speaking only } \\
\text { Spanish or other language) }\end{array}$ & 2.29 & .80 & 1.73 & .78 & $-.80^{* *}$ \\
\hline $\begin{array}{l}\text { Providers' negative attitudes towards } \\
\text { clients }\end{array}$ & 1.83 & .92 & 1.91 & 1.00 & .32 \\
\hline $\begin{array}{l}\text { Too few minority staff with whom } \\
\text { minority clients can identify }\end{array}$ & 1.83 & .76 & 1.78 & 1.06 & -.23 \\
\hline $\begin{array}{l}\text { Lack of appropriate interpreters } \\
\text { (having to use children, cleaning } \\
\text { staff, etc.) }\end{array}$ & 1.96 & .98 & 1.51 & .76 & $-2.01^{*}$ \\
\hline
\end{tabular}

delivery. As can be seen by the choices delineated in the Agency Cultural Competency Scale presented in Table 4, a higher score represents a greater degree of cultural awareness.

Independent two sample $t$-tests revealed a significant difference in perceptions. Administrators rated their agencies as more culturally competent than did staff $(M=3.72, S D .57$ vs. $M=3.18, S D=.90 ; t=-2.32, p<.05)$. Although on balance, both groups had very positive assessments of their agencies' level of cultural competency.

\section{DISCUSSION}

As an exploratory study examining providers in one geographic region, the results cannot be generalized to other contexts. Further, in light of the diversity intrinsic to the Hispanic classification (Castex, 1994), the applicability of results obtained from one group to others has been questioned (Austin \& Gilbert, 1989). Since a single homogeneous Hispano culture does not exist, replication with other Latino populations is clearly warranted.

Despite their diversity, there tends to be a number of shared cultural values among the discrete populations that comprise this group (Rodriguez \& Brindis, 1995). For example, familismo, the understanding that family is central to one's personal ontology, is a widely held value (Munoz, 1997; Rodriguez \& Brindis, 1995). Indeed, Madison Avenue's success in targeting the Latino market testifies to numerous points of congruence among discrete Hispanic groups. 


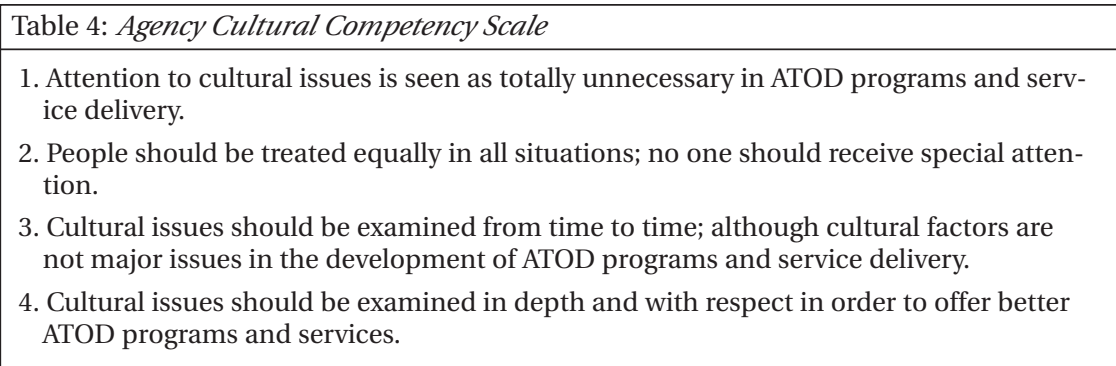

Furthermore, there is evidence that similarities may also exist in the purview of ATOD issues, as well. For instance, ATOD usage patterns show more symmetry than dissimilarity among diverse Hispanic populations (Warheit et al., 1996). In fact, Warheit, Vega, and Khoury (1996) have suggested the similarities are of such magnitude in this area that one can generally place Latinos in a single category without violating the integrity of their individuality.

Similarly, as noted in the introduction, widespread agreement exists that cultural sensitivity issues have broad applicability. In addition, acculturation tends to foster increased ATOD usage among numerous Latino subgroups. Indeed, in light of the acculturation effects seen in this geographic area, as well as other settings (Caetano, 1994; Casas et al., 1998; Deren et al., 1997; Warheit et al., 1996), this study may have particular salience for Hispanic-area ATOD agencies.

The high level of Hispano presence among providers was an unexpected finding. As mentioned above, the percentage of Latinos among administrators slightly exceeded the proportion within the surrounding population. This suggests acculturation may be a double-edged sword for Hispanics. While it may foster increased ATOD use, it may also facilitate higher levels of education and ATOD agency involvement.

Interestingly, Munoz (1997) has posited that the Hispano sense of familismo is not limited to the immediate family but is often generalized to the community. Correspondingly, individual identity development is intertwined with community development. In other words, as wholeness in the community is achieved, it is achieved in the individual. This dynamic may foster increased Hispanic involvement in ATOD agencies as a means to achieve community development. While some qualitative evidence supports this theory (Lazzari, Ford \& Haughey, 1996), further research with other samples of highly acculturated Latinos is required to confirm this hypothesis.

In terms of the cultural issues explored, providers perceived their agencies as needing only average amounts of training. There is little doubt that provider characteristics played a substantial role in this assessment. Providers were well educated, exhibited high levels of Spanish fluency, and perhaps most importantly, incorporated minority representation on a par with the surrounding community.

There was a noticeable pattern in both the training and barriers domains, although more pronounced in the latter. In questions that addressed cultural sensitivity issues at the interpersonal level, agencies were rated with more proficiency 
and barriers perceived to be less formidable. Conversely, broader, systemic, and macro issues were found at the other end of the continuum. Thus, for example, the lack of appropriate interpreters was the lowest obstacle impeding service utilization, while the lack of transportation systems was the highest.

Colon (1996) has suggested that the most important aspect of cultural sensitivity is the macro dimension (cf. Gant \& Gutierrez, 1996). Integrating the agency into the community through outreach, recruitment of community members, and inclusive decision making were understood to be essential to facilitating widespread Hispano use of services (Colon, 1996). Interestingly, even with the substantial numbers of locally "recruited" providers evident in this study, macro issues remained at the forefront of provider concern. It would seem that agency integration with the community is a prominent issue regardless of provider characteristics. In other words, recruiting local community members, even at the provider level, is only part of the solution. Macro outreaches, such as strategically located advertising agency services in Spanish, are a crucial element to enhancing service provision to Latinos (Bird et al., 1996).

Spanish language skills are essential in working with most Latino groups (Applewhite, Wong \& Daley, 1991; Ross, 1995; Santiago-Rivera, 1995). Provocatively, this was the only area where significant differences emerged between administrators and staff in the training and barrier domains. In both cases (lack of appropriate interpreters and clients' lack of proficiency in English), administrators, rather than staff, perceived a greater barrier existed.

Although no significant differences emerged between administrators and staff in terms of birthplace, ethnicity, or Spanish linguistic ability, staff were somewhat more likely to be born outside of New Mexico, to be Anglo, and to report less fluency in Spanish. These factors may have resulted in administrators' perceiving linguistic areas as more of an obstacle. The counterpoint to this explanation, however, is that no significant differences arose in other cultural areas. For example, assessments were essentially identical in other barriers, such as too few minority staff with whom minority clients can identify, and cultural differences between clients and providers. While more research is required, perhaps the most salient point is that both sets of providers perceived language to be a relatively minor hindrance to service.

Both administrators and staff had positive overall assessments of their agency's cultural competency in program development and service delivery. Although administrators held significantly higher perceptions of competency, this may have resulted from an enhanced sense of ownership toward their agencies. In spite of the differences, both sets of providers held very high opinions of their agency's attention to cultural issues.

\section{CONCLUSIONS}

This is the first empirical exploration through the eyes of administrators and staff of ATOD agency cultural sensitivity in an acculturated Hispanic area. No outstanding culturally related training needs appeared in administrators and staff perceptions. Concurrently, with the exception of clients' access to transportation, all culturally associated barriers received net negative scores, indicating that on 
balance, cultural barriers were not an overriding obstacle. This positive picture was confirmed by providers' high assessment of their agency's cultural competency in the purview of overall program development and service delivery. Additional corroboration is seen in the high degree of overlap between the results obtained in this study and the principles outlined by Gant and Gutierrez (1996) in terms of embodying their related concept of culturally sophisticated agencies.

The key factor that emerged in the substantial degree of cultural sensitivity can be traced to the high percentage of Hispano providers. Replication in alternate settings, including a qualitative component, may reveal that acculturation in tandem with a generalized sense of familismo fosters a high level of Latino presence among providers. Since minority providers play a critical role in facilitating a culturally sensitive environment, further empirical exploration is imperative.

\section{Acknowledgment}

The lead author would like to express his appreciation to Dr. David Arguello of New Mexico Highlands University for his encouragement and suggestions.

\section{References}

Applewhite, S. R., Wong, P., \& Daley, J. M. (1991). Service approaches and issues in Hispanic agencies. Administration and Policy in Mental Health, 19(1), 27-37.

Austin, G. A., \& Gilbert, J. M. (1989). Substance Abuse Among Latino Youth: Prevention Research Update, Number 3. The Western Center for drug-free schools and communities. Portland, Oregon: Northwest Regional Educational Laboratory.

Bird, J. A., Otero-Sabogal, R., Ha, N., \& McPhee, S. J. (1996). Tailoring lay health worker interventions for diverse cultures: Lessons learned from Vietnamese and Latina communities. Health Education Quarterly, 23[Suppl.], S105-S122.

Caetano, R. (1983). Drinking patterns and alcohol problems among Hispanics in the U.S.: A review. Drug and Alcohol Dependence, 12, 27-59.

Caetano, R. (1994). Drinking and alcohol-related problems among minority women. Alcohol Health \& Research World, 18(3), 233-241.

Carrasquillo, A. L. (1991). Hispanic children and youth in the United States: A resource guide. New York: Garland Publishing.

Casas, M. J., Bimbela, A., Corral, C. V., Yanez, I., Swaim, R. C., Wayman, J. C., \& Bates S. (1998). Cigarette and smokeless tobacco use among migrant and nonimmigrant Mexican American youth. Hispanic Journal of Behavioral Sciences, 20(1), 101-121.

Castex, G. M. (1994). Providing services to Hispanic/Latino populations: Profiles in diversity. Social Work, 39(3), 288-293.

Castro, F. (1997). Organizational capacity evaluation. Forms A \& B. Draft 2.0. Unpublished Survey Instruments. Arizona State University.

Colon, E. (1996). Program design and planning strategies in the delivery of culturally competent health and mental health prevention and treatment services to Latino communities. Journal of Multicultural Social Work, 4(4), 85-96.

Delgado, M., \& Tennstedt, S. (1997). Making the case for culturally appropriate community services: Puerto Rican elders and their caregivers. Health \& Social Work, 22(4), 246-255.

Delva-Tauili'ili, J. (1995). Assessment and prevention of aggressive behavior among youths of color: Integrating cultural and social factors. Social Work in Education, 17(2), 83-91.

Deren, S., Shedlin, M., Davis, W. R., Clatts, M. C., Balcorta, S., Beardsley, M. M., Sanchez, J., \& Jariais, D. D. (1997). Dominican, Mexican, and Puerto Rican prostitutes: Drug use and sexual behaviors. Hispanic Journal of Behavioral Sciences, 19(2), 202-213. 
Gant, L. M., \& Gutierrez, L. (1996). Effects of culturally sophisticated agencies on Latino social workers. Social Work, 41(6), 624-631.

Kahn, S. (1997). Leadership: Realizing concepts through creative process. Journal of Community Practice, 4(1), 109-136.

Lazzari, M. M., Ford, H. R., \& Haughey, K. J. (1996). Making a difference: Women of action in the community. Social Work, 41(2), 197-205.

Lewis, N. O. (Ed.). (1997). Minor under the influence: Underage drinking in New Mexico. Santa Fe, NM: New Mexico Traffic Safety Bureau.

Munoz, V. I. (1997). The passion that absorbs you: Latina/Latino identity development through community development. Education and Urban Society, 30(1), 41-57.

New Mexico Department of Health. (1996). 1994 New Mexico selected health statistics: Annual report. Santa Fe, NM: Bureau of Vital Records and Health Statistics.

Nuiry, O. E. (1997). Hispanics now a mass market in their own right. Public Relations Quarterly, 42, 28-29.

Parker, K. D., Weaver, G., \& Calhoun T. (1995). Predictors of alcohol and drug use: A multi-ethnic comparison. The Journal of Social Psychology, 135, 581-590.

Perez, S. M., \& De La Rosa Salazar, D. (1993). Economic, labor force, and social implications of Latino educational and population trends. Hispanic Journal of Behavioral Sciences, 15(2), 188-229.

Rodriguez, M. A., \& Brindis, C. D. (1995). Violence and Latino youth: Prevention and methodological issues. Public Health Reports, 110(3), 260-267.

Roslow, P., \& Nicholls, J. (1996). Targeting the Hispanic market: Comparative persuasion of TV commercials in Spanish and English. Journal of Advertising Research, 36(3), 67-77.

Ross, J. (1995). Who are they, where are they and how do we talk to them? Hispanic Americans. Hospitals and Health Networks, 69(19), 65-68.

Santiago-Rivera, A. L. (1995). Developing a culturally sensitive treatment modality for bilingual Spanishspeaking clients: Incorporating language and culture in counseling. Journal of Counseling and Development, 74(1), 12-17.

U. S. Bureau of the Census. (1998). 1990 US Census Online Database: C90STF1A. http://venus.census.gov.

U. S. Department of Health and Human Services. (1997). National household survey on drug abuse: Main findings 1995. Rockville MD: Substance Abuse and Mental Health Services Administration (SAMHSA), Office of Applied Studies.

Warheit, G. J., Vega, W. A., \& Khoury, E. L. (1996). A comparative analysis of cigarette, alcohol, and illicit drug use among an ethnically diverse sample of Hispanic, African American and non-Hispanic white adolescents. Journal of Drug Issues, 26, 901-922.

Weeks, M. R., Himmelgreen, D. A., Singer, M., Woolley, S., Romero-Daza, N., \& Grier, M. (1996). Community-based AIDS prevention: Preliminary outcomes of a program for African American and Latino injection drug users. Journal of Drug Issues, 26(3), 561-590.

Weil, M. (1996). Model development in community practice: An historical perspective. Journal of Community Practice, 3(3-4), 5-67.

Wright, J. D. (1994). Hispano forestry, land grants and the U.S. forest service in Northern New Mexico. Focus, 44, 10-14.

\section{Author's Note:}

Address correspondence to: David R. Hodge, Washington University, 1 Brookings Drive, Campus Box 1196, St. Louis, Missouri 63130-4899 USA. 